Research Article

\title{
Trajectory Data Compression Algorithm Based on Motion State Changing
}

\author{
Shuo Zhang, ${ }^{1}$ Jian Zhang, ${ }^{1}$ and Lin Qi ${ }^{1,2}$ \\ ${ }^{1}$ Beijing Information Science and Technology University, Beijing 100192, China \\ ${ }^{2}$ Beijing World Urban Circular Economy System (Industry) Collaborative Innovation Center, Beijing 100192, China \\ Correspondence should be addressed to Lin Qi; qilin@bistu.edu.cn
}

Received 22 December 2020; Revised 30 January 2021; Accepted 9 February 2021; Published 28 February 2021

Academic Editor: Rongwei Guo

Copyright (c) 2021 Shuo Zhang et al. This is an open access article distributed under the Creative Commons Attribution License, which permits unrestricted use, distribution, and reproduction in any medium, provided the original work is properly cited.

The trajectory information generated by the moving object plays an important role in studying the object movement. In this paper, a trajectory data compression algorithm based on the motion state changing is proposed to reduce trajectory data storage space and increase compression speed, which can accurately show the motion state and trajectory characteristics. This study has certain significance for the exploration of mass traffic data and the planning of traffic network. Combining the angle threshold with the velocity threshold of a moving object, the key data points are found and the redundant information is removed. Subsequently, the compressed trajectory is obtained. The experimental results show that the new algorithm can help to improve compression efficiency. The compressed trajectory has high similarity with the original trajectory in movement tendency.

\section{Introduction}

With the development of economy and technology, mobile devices and global positioning system are popular in various industries $[1,2]$. In particular, data collection and storage of trajectory information with the characteristics of time, location, speed, and direction are showing high-speed growth. Currently, how to compress and dispose the GPS data is becoming a hot spot. In 1973, David Douglas and Thomas Peucker presented a classical Douglas-Peucker algorithm to preferentially delete some points by means of information loss in iterative calculations. Afterwards, this algorithm was improved by many scholars. Hershberger John implemented the Douglas-Peucker algorithm for line simplification [3], and Jin et al. studied near-linear time approximation algorithms for curve simplification by reducing the time complexity [4]. In addition, Keogh proposed the Opening Window (OPW) algorithm based on the same algorithm in which the trajectory is simplified by iterative information loss. This algorithm cannot track information of the whole iteration; it is based on the concept of "open window" in which the algorithm "window" contains only a portion of track point iteration. Then, it keeps updating the track information in the "window" until the whole track simplification is completed. This algorithm can be synchronized with online track compression [5]. The sliding window algorithm [6] is similar to the open window algorithm, the main idea of which is to start from the track start, initialize a sliding window size of 1 , and gradually expand the window, thereby adding subsequent trajectory points. After connecting the first path point within the window and the last track point, the resulting segment is considered as segment approximation. Sliding window algorithm is used to calculate the approximate vertical line segment with the original track Euclidean distance; if the distance is less than the predetermined distance threshold, then continue to increase the sliding window. This process is repeated until the error is within the window. However, the abovementioned algorithm uses less track information using GPS time information. For this situation, Meratnia proposed a Top-Down Time Ratio algorithm [7]. This algorithm uses synchronous Euclidean distance (SEDm) in place of the vertical distance, which takes into account the time information from GPS track information. Coclite et al. [8] proposed using the road network of semantic information instead of track points to store compressed track objects and 
performed experimental studies in 2012. Yeh et al. used the oppressed road network link information in conjunction with information of the mobile objects' time to enter and leave the track [9]. This largely highly compressed the data storage. Threshold algorithm is another type of algorithm proposed by Al-Hussaeni et al. [10]. This algorithm is based on the moving object speed and direction at locus points to predict binding region threshold set at a point that may exist. Location prediction is decided by retaining or removing the points. Threshold algorithm deletes redundant indicator points based on speed and direction, and the algorithm takes into account the state trajectory, but the area to predict the next big point will possibly cause more points to delete. However, it can achieve a high compression ratio and has led to track trends and similar characteristics. Based on the above reasons, this paper constructs new indicators based on speed and angle, namely, the weighted combination of both thresholds to find the key points, and through statistical sampling to find the most appropriate threshold.

\section{Compression Algorithm Based on Angle Threshold}

The data used in this study are the taxi GPS positioning data of a Chinese megacity. The objective of the data compression algorithm is to show the trajectory form of taxi movement with the least GPS data.

Suppose there are $n$ taxi tracks. $X_{i}$ represents $i$ track, and each track has $m^{i}$ dots. A point of each track contains time, position, speed, and direction of rate in the GPS. $X_{i}^{j}$ represents $j$ point of $i$ track, where $j=1, \cdots, m_{i}$, and $X_{i}^{j}=\left(t_{i}^{j}, x_{i}^{j}, y_{i}^{j}, v_{i}^{j}, \theta_{i}^{j}\right)$. Five components of $X_{i}^{j}$ represent time, longitude, latitude, speed, and speed direction in $j$ point of $i$ track.

Due to fewer data points collected for each track, the data storage and calculating speed will be greatly affected. Therefore, the track can be compressed to find key points and remove redundant data on the premise that there is no loss of essential feature track [11-14]. In order to reduce the data amount, it is necessary to find the retained or removed data point indicators. From an intuitive point of view, when the velocity of a track point of direction change is large, it indicates that the vehicle is traveling or changing its track due to road change or incidental situation [15]. Thus, this paper first considers the speed direction (angle) as a screening data point indicator.

$\theta_{\alpha}$ is defined as the angle threshold, which is used to eliminate the trajectory points which are less than the angle threshold, and the scope of angle threshold is greater than 5 and less than 20. $\Delta \theta_{i}^{j, j+1}=\left|\theta_{i}^{j+1}-\theta_{i}^{j}\right|$ represents the absolute value of the change in the direction of the velocity values of two adjacent points in $i$ track. If $\Delta \theta_{i}^{j, j+1} \leq \theta_{\alpha}, X_{i}^{j}$ is deleted; if $\Delta \theta_{i}^{j, j+1}>\theta_{\alpha}, X_{i}^{j}$ is retained.

According to this principle, from the first point of a track, successive points will be tested backwards to get a compressed trajectory. In order to reflect the effect of compression, the compression ratio is defined as follows:

$$
Y_{i}^{\theta_{\alpha}}=\frac{m_{i}-m_{i}^{H}}{m_{i}},
$$

where $Y_{i}^{\theta \alpha}$ represents the compression rate of $i$ track with angle threshold $\theta_{\alpha}$ and $m_{i}$ and $m_{i}^{H}$ represent, respectively, the number of track points in $i$ track before and after compression [16].

Angle threshold $\theta_{\alpha}$ is defined, respectively, as $5,10,15$, and 20 in this paper. In nearly a month, running track (an example of the sample database shown in Table 1) of 2000 taxis in Beijing is verified, indicating that, with an increase in $\theta_{\alpha}$, the average compression rate increases.

With the increase in the angle threshold, the change of the average compression rate of 2000 taxis is shown in Figure 1. It can be seen from Figure 1 that the average compression rate is more than $45 \%$ and the time efficiency is improved greatly. However, since only the information point of view is considered, some points with an important feature of the information have been deleted so that the original motion trajectory trend has changed; for example, the license plate number "669148" taxi track is shown in Figure 2.

In Figure 2, with the angle threshold values of 5, 10, 15, and 20, four figures are shown before and after compression of the taxi track; the red trace is the original track and the blue trace is the track after compression. It is obvious that the significant changes in movement trend between points $\mathrm{A}$ and $\mathrm{B}$ before and after compression have taken place as shown in Figure 2; the main reason is that the locus points between points $\mathrm{A}$ and $\mathrm{B}$ are all deleted. In order to avoid the deletion of some key data points, the compression ratio is usually reduced. Therefore, we need to add a new threshold to filter the key data points that need to be retained. Thus, the information in the locus points contain the speed at different time periods; in addition, the size of the rate of change reflects whether there is traffic congestion or smooth, and it reveals the trajectory of internal features. If the speed is the only indicator, the situation with the angle threshold control of key points is bound to arise, which can get a higher compression ratio [17]. But it will lose some points that reflected track trends, and the characteristics of the track cannot be fully demonstrated by the rest of the points. For these reasons, this paper, used the two indicators angle and speed as the key points of track [18-20].

\section{Compression Algorithm Based on Angle and Speed Threshold}

Speed threshold is defined as $v_{\alpha}$, which is used to eliminate the trajectory points which are less than the speed threshold, and the scope of the speed threshold is greater than 5 and less than $20 . \Delta v_{i}^{j, j+1}=\left|v_{i}^{j+1}-v_{i}^{j}\right|$ represents the absolute value of 
TABLE 1: Example of the sample database.

\begin{tabular}{|c|c|c|c|c|c|}
\hline Plate number & Time & Longitude & Latitude & Speed & Direction (angle) \\
\hline 669148 & 20121130044746 & 116.134 & 39.642 & 0 & 194 \\
\hline 669148 & 20121130001657 & 116.373 & 39.987 & 25 & 184 \\
\hline 669148 & 20121130001751 & 116.373 & 39.984 & 54 & 166 \\
\hline 669148 & 20121130001845 & 116.374 & 39.98 & 47 & 164 \\
\hline 669148 & 20121130001940 & 116.375 & 39.976 & 0 & 168 \\
\hline 669148 & 20121130002033 & 116.375 & 39.974 & 43 & 186 \\
\hline 669148 & 20121130002126 & 116.374 & 39.967 & 23 & 268 \\
\hline 669148 & 20121130002219 & 116.365 & 39.967 & 60 & 268 \\
\hline
\end{tabular}

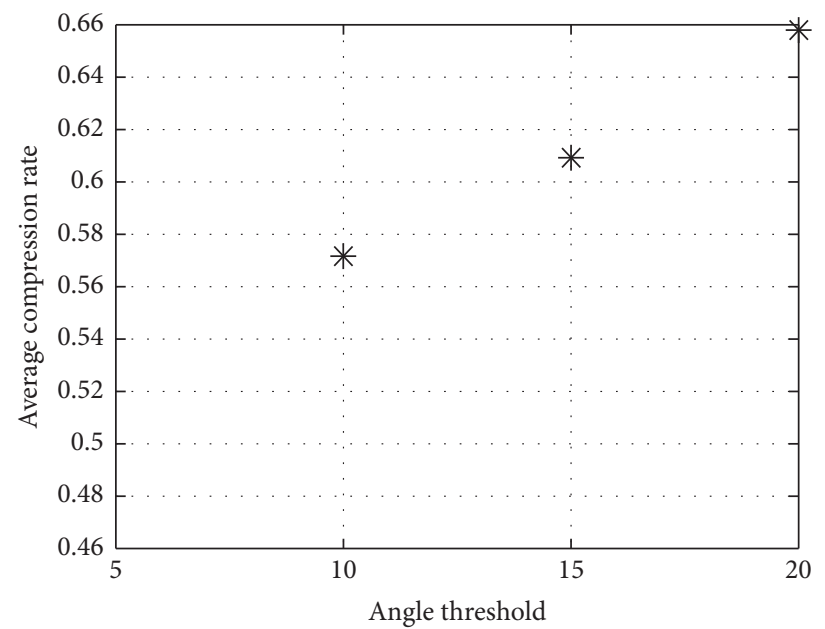

Figure 1: The trend of the average compression rate with the angle threshold.

the change in the speed of the velocity values of two adjacent points in $i$ track. These points that meet the limit of angle threshold and speed threshold are deleted, namely, if $\Delta \theta_{i}^{j, j+1} \leq \theta_{\alpha}$ and $\Delta v_{i}^{j, j+1} \leq v_{\alpha}, X_{i}^{j}$ is deleted, or else, $X_{i}^{j}$ is retained.

According to this principle, from the first point of a track, successive points will be tested backwards to get a compressed trajectory. The track is still referred to be $X_{i}^{H}$ in the case not to cause confusion. Simultaneously, the compression ratio is defined as follows:

$$
Y_{i}^{\theta_{\alpha}, v_{\alpha}}=\frac{m_{i}-m_{i}^{H}}{m_{i}},
$$

where $m_{i}$ is as defined above and $m_{i}^{H}$ is the number of compressed track points in the angle and speed threshold control.

Angle threshold $\theta_{\alpha}$ is defined, respectively, as 5, 10, 15, and 20 , and the speed threshold $v_{\alpha}$ is defined, respectively, as $5,10,15,20,25$, and 30 in this paper. Thus, 30 combinations are obtained.

As shown in Figure 3, track trends of A and B points before and after compression are well preserved in this method. In Figure 3, tracks are shown in the last small picture with grid before and after compression when $\theta_{\alpha}=25$ and $v_{\alpha}=30$. In addition, two encircled points, respectively, in Figure 3 are points $A$ and $B$ in Figure 2. It is very intuitive that motion trends of point $A$ and point $B$ were retained well even in the larger threshold, but the compression ratio of this algorithm is lower.
The average compression ratio of 2000 taxi track trends with the angle and speed threshold values at the same time is shown in Figure 4 . Thus, the highest value of compression ratio is about $50 \%$ and the lowest value is only about $20 \%$. We have paid more attention to keeping contrail features and being stringent to take data points so that a few points meet the speed and angle threshold. Besides, it leads to a situation that the similarity of the track shape is higher, but the compression rate is lower. When the angle and velocity threshold values change, the compression ratio also changes in size as shown in Figure 4.

\section{Compression Algorithm of Trajectory Data Based on Motion State Change}

Assuming a new index, the weighted threshold value for velocity and angle, so as to obtain higher compression ratio and retain important information after compression [21], the index is marked as $\theta v_{\alpha}$. Making $\Delta \theta v_{i}^{j, j+1}=\alpha_{1} \Delta \theta_{i}^{j, j+1}+$ $\alpha_{2} \Delta v_{i}^{j, j+1}$, where $\Delta \theta_{i}^{j, j+1}$ and $\alpha_{2}$ are the weights of $\Delta \theta_{i}^{j, j+1}$ and $\Delta v_{i}^{j . j+1}$, respectively. If $\Delta \theta v_{i}^{j, j+1} \leq \theta v_{\alpha}, X_{i}^{j}$ is deleted; else, $X_{i}^{j}$ is retained.

Using the mathematical model established above, each data point of the vehicle trajectory data is screened in turn, and the key data points left by the screening are used to form the compressed vehicle trajectory. Without a disordered case, mark the compressed trajectory as $X_{i}^{H}$, and the 


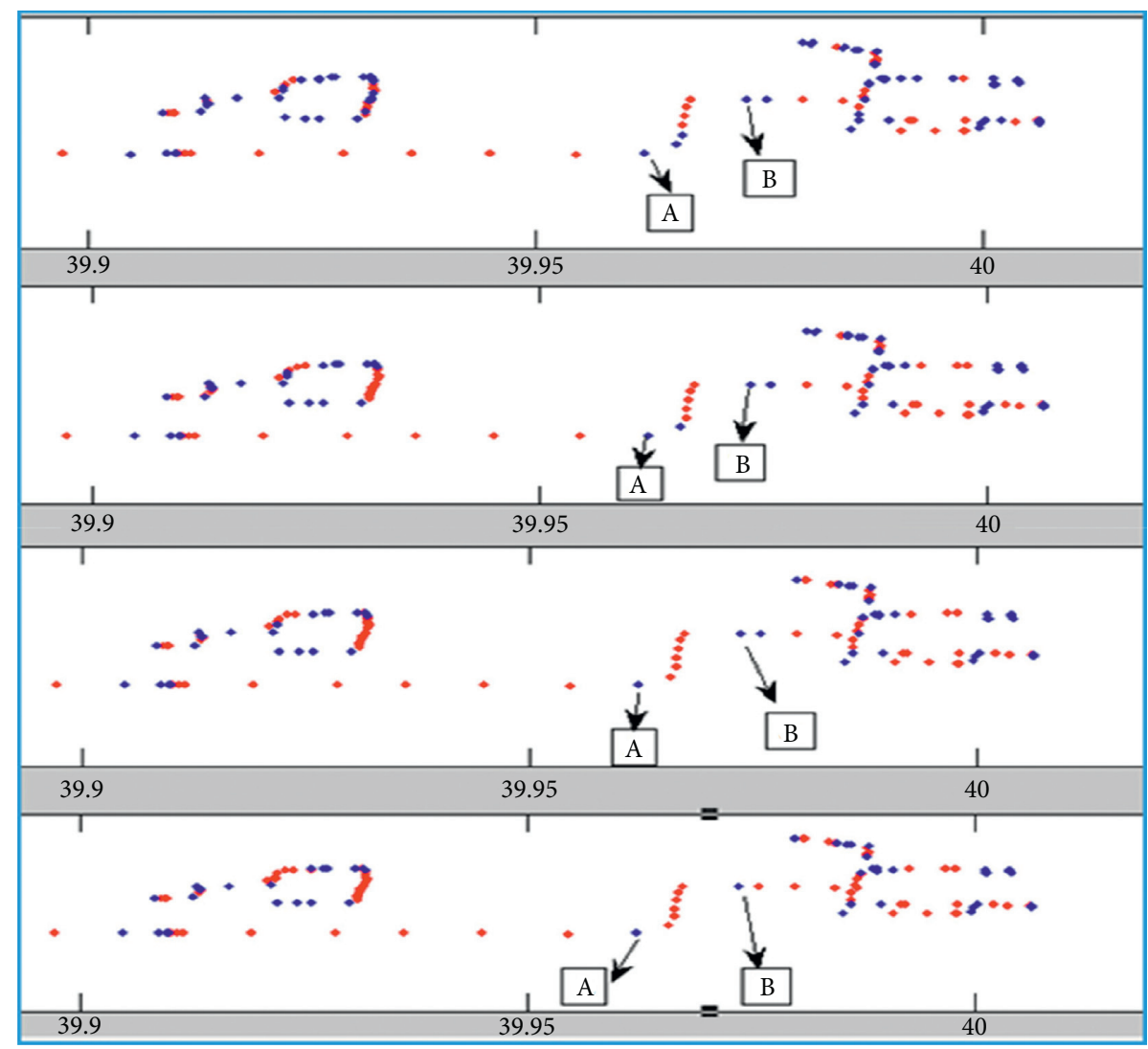

FIGURE 2: Trajectory map before and after compression at different angles.

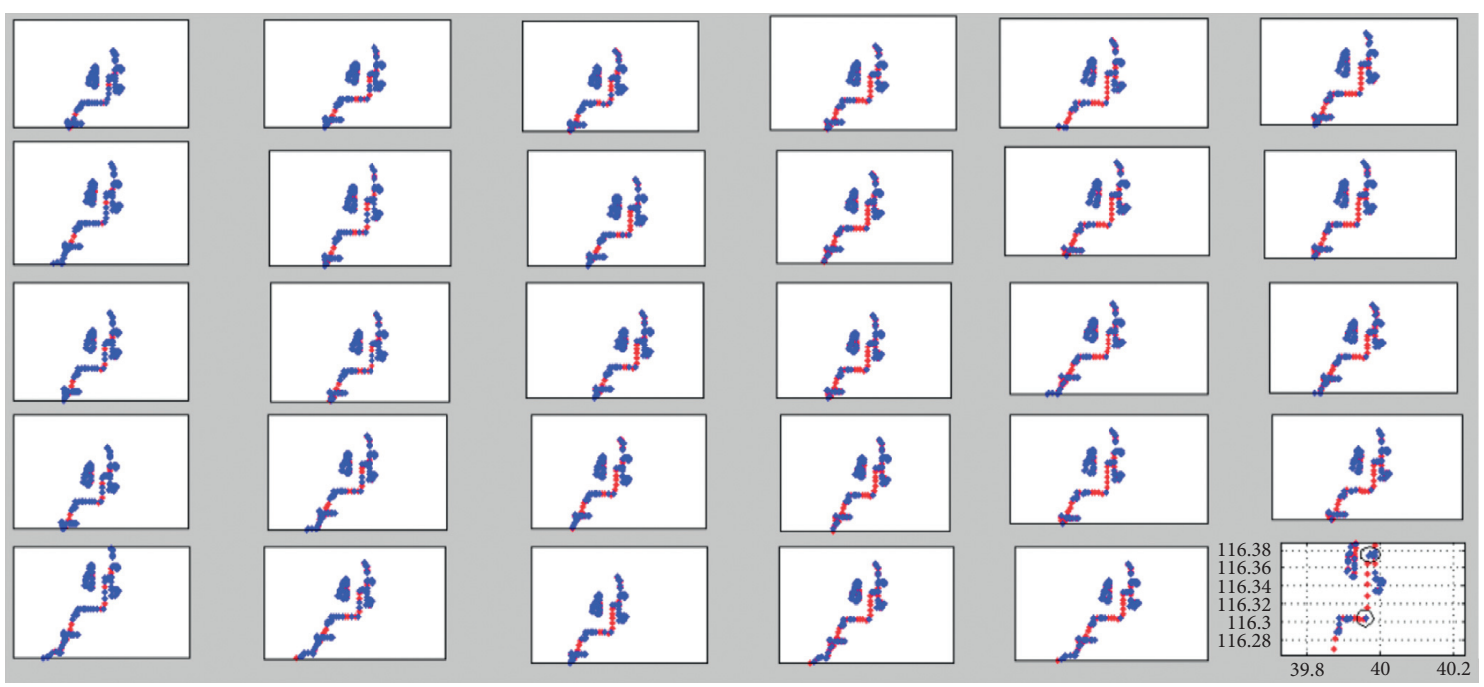

FIGURE 3: Trajectory map before and after compression at different angles and speeds.

compression ratio is defined as $Y_{i}^{\theta v_{\alpha}}=\left(\left(m_{i}-m_{i}^{H}\right) / m_{i}\right)$, but $m_{i}$ as primary. Under the control of the weighted threshold value for velocity and angle, $m_{i}^{H}$ is the number of trajectory points compressed [22].

Reduced trajectory was not only considering efficiency, but it was also guaranteeing the compressed trajectory as well as the original trajectory. In order to reflect the similarity of the two trajectories before and after compression, the diversity of the two trajectories is defined. $D_{i}$ represents the absolute difference of $i$ track before and after compression. Owing to the concern about kinetic trend changes before and after compression [23], area of the shadow that the compression trajectory and the original trajectory are surrounded by was not too large, as shown in Figure 5.

In Figure 5, the red line represents the point of original trajectory data and the blue line represents the point of 


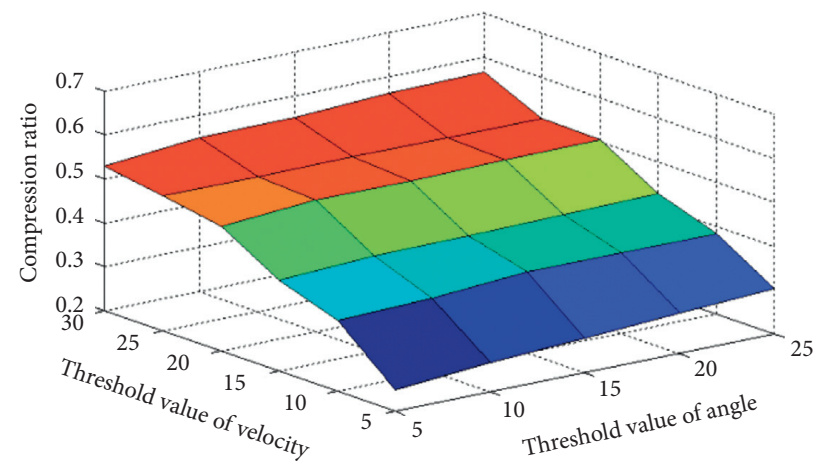

Figure 4: The change of angle threshold and velocity threshold leads to the change trend of compression ratio.

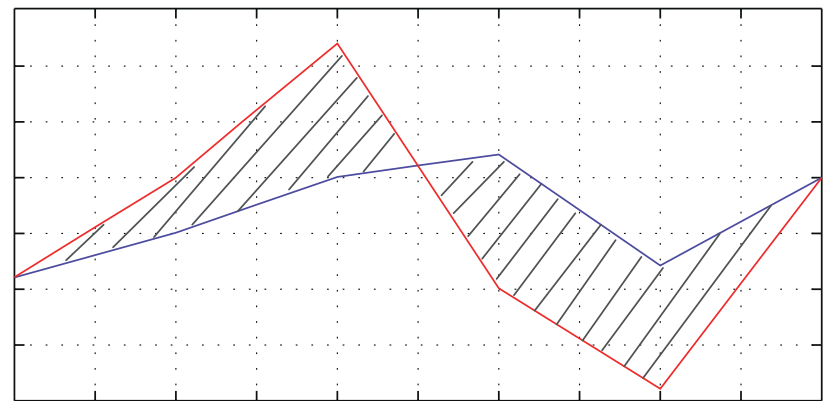

FIgURE 5: The area of the shadow that the compression trajectory and the original trajectory are surrounded by.

TABLE 2: Average compression ratio and average relative diversity of different threshold values and weights.

\begin{tabular}{|c|c|c|c|c|c|c|}
\hline $\begin{array}{l}\text { Threshold } \\
\text { value }\end{array}$ & $\begin{array}{c}\text { Weight } \\
1\end{array}$ & $\begin{array}{l}\text { Weight } \\
2\end{array}$ & $\begin{array}{l}\text { The average } \\
\text { compression ratio }\end{array}$ & $\begin{array}{l}\text { The average } \\
\text { relative diversity }\end{array}$ & $\begin{array}{l}1-\text { the average } \\
\text { relative diversity }\end{array}$ & $\begin{array}{l}P=1-\text { the average compression } \\
\text { ratio }+(1-\text { the average relative diversity })\end{array}$ \\
\hline \multirow{9}{*}{10} & 0.1 & 0.9 & 0.4398 & 0.24087042 & 0.75912958 & 1.198929583 \\
\hline & 0.2 & 0.8 & 0.40601504 & 0.00037894 & 0.99962106 & 1.405636094 \\
\hline & 0.3 & 0.7 & 0.39849624 & 0.00040007 & 0.99959993 & 1.398096167 \\
\hline & 0.4 & 0.6 & 0.39097744 & 0.00040175 & 0.99959825 & 1.390575697 \\
\hline & 0.5 & 0.5 & 0.41353384 & 0.00040171 & 0.99959829 & 1.413132124 \\
\hline & 0.6 & 0.4 & 0.42481203 & 0.00034475 & 0.99965525 & 1.424467279 \\
\hline & 0.7 & 0.3 & 0.33082707 & 0.00031865 & 0.99968135 & 1.330508419 \\
\hline & 0.8 & 0.2 & 0.4962406 & 0.09109596 & 0.90890404 & 1.405144641 \\
\hline & 0.9 & 0.1 & 0.4962406 & 0.09514899 & 0.90485101 & 1.401091612 \\
\hline \multirow{9}{*}{15} & 0.1 & 0.9 & 0.5 & 0.28109667 & 0.71890333 & 1.218903331 \\
\hline & 0.2 & 0.8 & 0.48872181 & 0.00076605 & 0.99923395 & 1.487955751 \\
\hline & 0.3 & 0.7 & 0.5112782 & 0.0004619 & 0.9995381 & 1.510816294 \\
\hline & 0.4 & 0.6 & 0.5037594 & 0.0004616 & 0.99953841 & 1.503297804 \\
\hline & 0.5 & 0.5 & 0.52255639 & 0.00069379 & 0.99930621 & 1.521862599 \\
\hline & 0.6 & 0.4 & 0.54887218 & 0.09412948 & 0.90587052 & 1.454742699 \\
\hline & 0.7 & 0.3 & 0.39473684 & 0.00040175 & 0.99959825 & 1.39433509 \\
\hline & 0.8 & 0.2 & 0.59398496 & 0.09527842 & 0.90472158 & 1.49870654 \\
\hline & 0.9 & 0.1 & 0.59398496 & 0.09526771 & 0.90473229 & 1.498717254 \\
\hline \multirow{9}{*}{20} & 0.1 & 0.9 & 0.5977 & 0.28465153 & 0.71534847 & 1.313048474 \\
\hline & 0.2 & 0.8 & 0.57894737 & 0.24425466 & 0.75574534 & 1.33469271 \\
\hline & 0.3 & 0.7 & 0.54511278 & 0.00076575 & 0.99923425 & 1.544347035 \\
\hline & 0.4 & 0.6 & 0.57894737 & 0.09443527 & 0.90556473 & 1.484512094 \\
\hline & 0.5 & 0.5 & 0.60150376 & 0.09413002 & 0.90586998 & 1.507373736 \\
\hline & 0.6 & 0.4 & 0.62030075 & 0.09418688 & 0.90581312 & 1.526113872 \\
\hline & 0.7 & 0.3 & 0.4962406 & 0.00046169 & 0.99953831 & 1.49577891 \\
\hline & 0.8 & 0.2 & 0.64285714 & 0.09532709 & 0.90467291 & 1.547530049 \\
\hline & 0.9 & 0.1 & 0.64285714 & 0.09532786 & 0.90467214 & 1.547529287 \\
\hline
\end{tabular}


TABLE 2: Continued.

\begin{tabular}{lcccccc}
\hline $\begin{array}{l}\text { Threshold } \\
\text { value }\end{array}$ & $\begin{array}{c}\text { Weight } \\
1\end{array}$ & $\begin{array}{c}\text { Weight } \\
2\end{array}$ & $\begin{array}{c}\text { The average } \\
\text { compression ratio }\end{array}$ & $\begin{array}{c}\text { The average } \\
\text { relative diversity }\end{array}$ & $\begin{array}{c}1-\text { the average } \\
\text { relative diversity }\end{array}$ & $\begin{array}{c}P=1-\text { the average compression } \\
\text { ratio }+(1-\text { the average relative diversity })\end{array}$ \\
\hline & 0.1 & 0.9 & 0.6429 & 0.32357906 & 0.67642094 & 1.319320939 \\
& 0.2 & 0.8 & 0.62781955 & 0.29011079 & 0.70988921 & 1.337708762 \\
& 0.3 & 0.7 & 0.62406015 & 0.09759624 & 0.90240376 & 1.526463914 \\
25 & 0.4 & 0.6 & 0.64661654 & 0.09760408 & 0.90239592 & 1.549012461 \\
& 0.5 & 0.5 & 0.67293233 & 0.09854359 & 0.90145641 & 1.574388742 \\
& 0.6 & 0.4 & 0.67293233 & 0.09635091 & 0.90364909 & 1.525621997 \\
& 0.7 & 0.3 & 0.52631579 & 0.00069379 & 0.99930621 & 1.586400983 \\
& 0.8 & 0.2 & 0.68421053 & 0.09780954 & 0.90219046 & 1.582227325 \\
0.9 & 0.1 & 0.68421053 & 0.1019832 & 0.8980168 & 1.16812343 \\
& 0.1 & 0.9 & 0.7406 & 0.57247657 & 0.42752343 & 1.374235114 \\
& 0.2 & 0.8 & 0.70300752 & 0.38568571 & 0.61431429 & 1.596949488 \\
& 0.3 & 0.7 & 0.71428571 & 0.3400506 & 0.6599494 & 1.592093231 \\
& 0.4 & 0.6 & 0.69548872 & 0.09853923 & 0.90146077 & 1.615659076 \\
& 0.5 & 0.5 & 0.69172932 & 0.09963609 & 0.90036391 & 1.492336042 \\
0.6 & 0.4 & 0.71804511 & 0.10238604 & 0.89761396 & 1.597154926 \\
& 0.7 & 0.3 & 0.58646617 & 0.09413012 & 0.90586988 & 1.597155012 \\
\hline
\end{tabular}

compressed trajectory data. If key points are selected properly, the shaded area would be as small as possible. Based on the above considerations, define

$$
\begin{aligned}
D_{i}= & \left|\sum_{j-1}^{m_{i}-1}\left(y_{j}+y_{j+1}\right)\right| x_{j+1}-x_{j} \mid \\
& -\sum_{k-1}^{m_{i}^{H}-1}\left(y_{k}^{H}+y_{k+1}^{H}\right)\left|x_{k+1}^{H}-x_{k}^{H}\right|=S_{i}-S_{i}^{H},
\end{aligned}
$$

where $S_{i}$ is the area of the original trajectory surrounding the horizontal axis and $S_{i}^{H}$ is the area of the compressed trajectory surrounding the horizontal axis.

Because of the different lengths of the original trajectory, the difference between the two tracks is called relative diversity, to be called $D_{i}^{\prime}=\left(D_{i} / S_{i}\right)$. Taking $\theta v_{\alpha}=10,15,20,25$, and 30 and $\left(\alpha_{1}, \alpha_{2}\right)=(0.1,0.9),(0.2,0.8),(0.3,0.7),(0.4,0.6), \ldots,(0.9$, 0.1 ) as weights, the experiments of 2000 trajectories were run. According to various values, the average trajectory compression ratio was calculated and the average relative diversity was reckoned. The group that had a high average compression rate and a low average relative diversity as final thresholds and last weights was selected. Test results are shown in Table 2. In order to conveniently determine the threshold value and weights, choose by the size of $P=1$-the average compression ratio $+(1-$ the average relative diversity). As is shown in Table 1 , when $\theta v_{\alpha}=30, P$-values for different situations are maximum. So 30 and $0.6,0.4$ were determined as threshold value and weights. At the moment, the average compression ratio was greater than $71 \%$, the average relative diversity was only $10 \%$, and the similarity of the original trajectory and the compressed trajectory was approximately $90 \%$.

\section{Conclusions and Discussion}

Exploring the law of vehicle trajectory data can uncover some important road network information, which can provide effective decisions and suggestions for reducing road congestion and planning of traffic routes. To explore the value of object trajectory information, we study a vehicle trajectory data compression algorithm based on the change of motion state. The advantage of the algorithm is that the vehicle trajectory data compression rate is high, and the vehicle motion state and trajectory characteristics can be displayed as accurately as possible. In the research process of the algorithm, we use the GPS data of taxi track to do a lot of experiments to explore the impact of speed threshold and angle threshold on the track data compression rate. According to the experimental results, we propose a threshold combination algorithm, which improves the data compression rate by changing the threshold parameters and makes the compressed data clearly show the characteristics of vehicle trajectory. Reference values are as follows:

(1) Angle threshold is defined, respectively, as 5, 10, 15, and 20 in this paper. In nearly a month, running track of 2000 taxis in Beijing is verified, when the average compression rate is more than $45 \%$. However, some points with an important feature of the information have been deleted so that the original motion trajectory trend has changed. Obviously, it is unscientific to only use the angle threshold to screen out the key data points in the vehicle trajectory; therefore, we need to add a new threshold to screen out the key data points that need to be retained. Thus, the information about the locus points contains the 
speed at different times; in addition, the size of the rate of change reflects whether there is traffic congestion or smooth, and it reveals the trajectory of internal features. If the speed is the only indicator, the situation with the angle threshold control of key points is bound to arise, which can get a higher compression ratio. But it will lose some points that reflected track trends, so that the characteristics of the track cannot be fully demonstrated by the rest of the points.

(2) Angle threshold is defined, respectively, as 5, 10, 15, and 20, and speed threshold is defined, respectively, as $5,10,15,20$, and 25 ; the highest value of compression ratio is about $50 \%$, and the lowest value is only about $20 \%$. We have paid more attention to keeping contrail features and being stringent to take data points so that a few points meet the speed and angle threshold. Besides, it leads to a situation that the similarity of the track shape is higher, but the compression rate is lower.

(3) Using speed threshold and angle threshold to set a new index so as to obtain higher compression ratio and retain important information after compression, the index setting and parameter selection can be obtained through a large number of experiments. Angle threshold and velocity threshold are controlled by parameters so that reduced trajectory was not only considering efficiency but also guaranteeing the compressed trajectory as well as the original trajectory. Finally, the vehicle trajectories before and after compression are presented and similarity analysis is carried out.

\section{Data Availability}

The data presented in this study are available upon request from the corresponding author.

\section{Conflicts of Interest}

The authors declare that they have no conflicts of interest regarding the publication of this paper.

\section{Acknowledgments}

This research was funded by the Project of Promoting the Connotative Development of Beijing Information Science and Technology University (521201090A).

\section{References}

[1] C. L. Meehan, D. V. Cacciola, and M. Khosravi, "Monitoring field lift thickness using compaction equipment instrumented with global positioning system (GPS) technology," Geotechnical Testing Journal, vol. 36, no. 5, pp. 755-767, 2013.

[2] S. Khan, P. Maini, and K. Thanasupsin, "Car-following and collision constraint models for uninterrupted traffic: reexamination using high-precision global positioning system data," Transportation Research Record: Journal of the Transportation Research Board, vol. 1710, no. 1, pp. 37-46, 2000.
[3] L. Zhao and G. Shi, "A trajectory clustering method based on Douglas-Peucker compression and density for marine traffic pattern recognition," Ocean Engineering, vol. 172, pp. 456467, 2019.

[4] K. Jin, J. Li, H. Wang, B. Zhang, and N. Zhang, "Near-linear time approximation schemes for geometric maximum coverage," Theoretical Computer Science, vol. 725, pp. 64-78, 2018.

[5] S. Khan, P. Maini, and K. Thanasupsin, "Stability control and turning algorithm of an alpine skiing robot," Sensors, vol. 19, no. 17, 2019.

[6] W. Song, R. Zhang, Y. Yao, Y. Liu, and Y. Hu, "PPP sliding window algorithm and its application in deformation monitoring," Scientific Reports, vol. 6, no. 1, pp. 149-158, 2016.

[7] H. Alborzi and H. Samet, "Execution time analysis of a topdown R-tree construction algorithm," Information Processing Letters, vol. 101, no. 1, pp. 6-12, 2006.

[8] G. M. Coclite, M. Garavello, and B. Piccoli, "Traffic flow on a road network," SIAM Journal on Mathematical Analysis, vol. 36, no. 6, pp. 1862-1886, 2006.

[9] A. G. O. Yeh, T. Zhong, and Y. Yue, "Hierarchical polygonization for generating and updating lane-based road network information for navigation from road markings," International Journal of Geographical Information Science, vol. 29, no. 9, pp. 1509-1533, 2015.

[10] K. Al-Hussaeni, B. C. M. Fung, F. Iqbal, G. G. Dagher SafePath, and E. G. Park, "SafePath: differentially-private publishing of passenger trajectories in transportation systems," Computer Networks, vol. 143, pp. 126-139, 2018.

[11] R. Mahbubani, P. Schwaller, and J. Zurita, "Erratum to: closing the window for compressed dark sectors with disappearing charged tracks," Journal of High Energy Physics, vol. 2017, no. 6, pp. 1-22, 2017.

[12] C. L. Meehan, D. V. Cacciola, and M. Khosravi, "Robust multi-scale ship tracking via multiple compressed features fusion," Signal Processing: Image Communication, vol. 31, pp. 76-85, 2015.

[13] X. Yi, R. Guo, and Y. Qi, "Stabilization of chaotic systems with both uncertainty and disturbance by the UDE-based control method," IEEE Access, vol. 8, no. 1, pp. 62471-62477, 2020.

[14] X.-J. Shen, L. Mu, Z. Li, H.-X. Wu, J.-P. Gou, and X. Chen, "Large-scale support vector machine classification with redundant data reduction," Neurocomputing, vol. 172, pp. 189-197, 2015.

[15] H. Mahdin and J. Abawajy, "An approach for removing redundant data from RFID data streams," Sensors, vol. 11, no. 10, pp. 9863-9877, 2011.

[16] E. Kotter, A. Roesner, J. Torsten Winterer et al., "Evaluation of Lossy data compression of chest X-rays: a receiver operating characteristic study," Investigative Radiology, vol. 38, no. 5, pp. 243-249, 2003.

[17] N. K. Kasumov, "Data positioning as an instrument of constructive data compression," Automatic Control and Computer Sciences, vol. 46, no. 5, pp. 223-231, 2012.

[18] L. Liu, B. Li, and R. Guo, "Consensus control for networked manipulators with switched parameters and topologies," IEEE Access, vol. 9, pp. 9209-9217, 2021.

[19] R. Xu and F. Zhang, " $\varepsilon$-Nash mean-field games for general linear-quadratic systems with applications," Automatica, vol. 114, pp. 1-6, 2020.

[20] W. Wang, Y. He, J. Liu, and S. Gombault, "Constructing important features from massive network traffic for lightweight intrusion detection," IET Information Security, vol. 9, no. 6, pp. 374-379, 2015. 
[21] T. Hou, Y. Liu, and F. Deng, "Finite horizon H2/Hoo control for SDEs with infinite Markovian jumps," Nonlinear Analysis: Hybrid Systems, vol. 34, pp. 108-120, 2019.

[22] S. Ke, J. Gong, S. Li, Q. Zhu, X. Liu, and Y. Zhang, "A hybrid spatio-temporal data indexing method for trajectory databases," Sensors, vol. 14, no. 7, pp. 1290-3005, 2014.

[23] L. Chen, M. Lv, Ye Qian, G. Chen, and J. Woodward, "A personal route prediction system based on trajectory data mining," Information Sciences, vol. 181, no. 7, pp. 1264-1284, 2010 . 